\title{
Décrochage scolaire des jeunes filles au Mali : contraintes et défis
}

\author{
Seydou LOUA ${ }^{a^{*}}$ \\ ${ }^{a}$ Université des Lettres et des Sciences Humaines de Bamako (ULSHB), Mali \\ * seydouloua@yahoo.fr
}

\section{Résumé}

Le travail s'articule autour des questions suivantes : Quelles sont les pesanteurs socioculturelles qui expliquent le décrochage scolaire rapide des filles au Mali ? Quelles alternatives d'amélioration ont été mises en place ? Quelles sont les limites des politiques publiques jusqu'ici engagées ? L'objectif visé est pluriel car, il s'agit entre autres, d'analyser la situation scolaire des jeunes filles maliennes, de saisir les raisons de leur décrochage, de décrypter les politiques publiques en la matière et de dégager quelques perspectives d'amélioration.

Mots clés : abandon, décrochage, jeune fille, politiques publiques, scolarisation, tradition

\section{School dropout of young girls in Mali: constraints and challenges}

\begin{abstract}
The work revolves around the following questions: What are the socio-cultural constraints that explain the rapid school dropout of girls in Mali? What improvement alternatives have been put in place? What are the limits of the public policies undertaken so far? The objective is plural because it involves, among other things, analyzing the educational situation of young Malian girls, understanding the reasons for their dropout, deciphering public policies in this area and identifying some prospects for improvement.
\end{abstract}

Keywords: dropout, young girl, public policies, schooling, tradition 
The Journal of Quality in Education (JoQiE) Vol.11, N²17, May 2021

\section{Introduction}

La situation scolaire de la fille au Mali a toujours occupé une place importante dans les politiques de développement. Bien que des avancées notoires soient à signaler comme l'amélioration de leurs taux de scolarisation, les jeunes filles abandonnent l'école toujours plus rapidement et massivement que leurs camarades garçons. Dans les sociétés africaines en général, les hommes et les femmes sont caractérisés par des différences au niveau de la division du travail (à la maison comme au service), l'exercice du pouvoir (coutumier et politique est exclusivement masculin dont la gouvernance féminine est perçue comme une anomalie passagère), la répartition des revenus, le pouvoir de décision. Cette situation joue négativement sur la scolarité de la jeune fille, qui est non seulement sous scolarisée, mais décroche plus facilement que le garçon. Dans le rapport du Programme d'analyse des systèmes éducatifs de la Confemen (Pasec, 2015), il est dit que «lorsque les différences liées au genre existent au niveau de l'école, elles sont toujours en faveur des garçons ».

En 2016 le taux net de scolarisation au primaire au Mali était de 61,4\% pour les garçons et 58,9\% pour les filles (EMOP, 2017). A la même période, selon les statistiques de l'UNICEF de 2016, le taux d'achèvement de l'enseignement primaire était de $47 \%$ pour les garçons et de $39 \%$ pour les filles. Les disparités sont encore plus grandes quand on compare les milieux ruraux des milieux urbains. Plusieurs raisons peuvent expliquer les difficultés et le décrochage scolaire de la jeune fille, notamment le poids de la tradition, l'interprétation tronquée ou falsifiée des textes sacrés, surtout musulmans, la pauvreté, le mariage et la grossesse précoce, etc. Pour expliquer le décrochage scolaire des filles, Marianne Ophéim (2000) cite des facteurs comme l'éloignement de l'école, certaines valeurs familiales africaines, les travaux domestiques, etc. Dans un article, (Loua, 2018), il est noté que tous ces facteurs 
énumérés par Opheim, restent d'actualité encore aujourd'hui dans la société malienne, malgré les avancées enregistrées par les gouvernements successifs en matière de scolarisation et de maintien des filles à l'école.

\section{Situation scolaire des jeunes filles maliennes}

Le Mali a connu la colonisation dans les années 1800 et la première école française sur son sol en 1886, une nouvelle forme d'éducation avec une langue différente et des principes venus d'ailleurs. Cette école française a été dans un premier temps, redoutée et rejetée par la population malienne, ce qui explique le caractère obligatoire de la scolarisation à l'époque. Le caractère discriminatoire de la scolarisation concernant les filles a commencé depuis cette époque, car le colonisateur ne scolarisait que les fils de chefs coutumiers afin d'asseoir dans la durée sa domination sur toute la communauté. Le gouverneur général Ernest Roume, gouverneur général de l’AOF de 1902 à 1907, expliquait les raisons de cette sélection en ces termes :

Choisissons judicieusement cette minorité, considérons l'instruction comme une chose précieuse qu'on ne distribue qu'à bon escient et limitons-en les bienfaits...

Choisissons nos élèves tout d'abord parmi les fils de chefs et de notables car la société indigène est très hiérarchisée. (Moumouni, 1998)

À l'indépendance, en 1960, le taux de scolarisation au Mali était entre 8 et $10 \%$ (Diambomba, 1980), ce qui a conduit le Mali, à partir de 1962, d'effectuer des réformes et innovations structurelles et pédagogiques dans son système éducatif. L'amélioration de la scolarisation des filles était considérée comme une priorité dans toutes ces réformes. La réforme de 1962, avec le début du processus de massification, le programme décennal de développement de l'éducation (PRODEC), qui a pris fin en 2012, avec son objectif « Un village, une école et/ou un centre d'éducation pour le développement », la Nouvelle Ecole Fondamentale, la multiplication des écoles privées, 
ont permis d'améliorer la scolarisation des filles. Dans un article (Loua, 2018), il est à noter que :

L'accent sur l'éducation des filles en Afrique, auquel le Mali a pris part, s'est traduit par un certain nombre d'engagements essentiels, dont la Plateforme d'action de Beijing, en 1995, qui était un programme ambitieux pour l'autonomisation des femmes, impliquant des représentants de 189 gouvernements, et le Cadre d'action de Dakar (adopté par le Forum Mondial sur l'Education, en avril 2000) en faveur d'une Éducation pour tous et les objectifs du Millénaire pour le développement. En Afrique, la décennie 2006-2015 a été marquée par des engagements et des objectifs spécifiques, comme l'élimination des disparités liées au genre, afin de garantir l'égalité, l'autonomisation des filles et des femmes par le biais de l'éducation. La biennale de l'Association pour le développement de l'éducation en Afrique (ADEA), en 2008, a conforté cet état de fait, en faisant de la transition de l'éducation de base vers les niveaux supérieurs une priorité et en proposant plus d'enseignants de sexe féminin au niveau post-primaire. Les congrès internationaux des ministres de l'éducation, tel que celui de la Communauté économique des États de l'Afrique de l'Ouest (CEDEAO), en 2004, ont donné la priorité aux engagements pour l'éducation des filles.

Malgré les mesures prises par les autorités politiques, scolaires et les partenaires, qui ont favorisé une certaine parité entre garçons et filles au niveau de la scolarisation, beaucoup reste à faire pour réduire le décrochage scolaire des filles et les maintenir à l'école aussi longtemps que possible au même titre que les garçons. Le tableau suivant fait état de quelques statistiques de la cellule de planification et de statistique du Ministère de l'éducation nationale 
Tableau 1 : Taux de scolarisation et d'achèvement de l'éducation de base de 2014 à 2017

\begin{tabular}{|c|c|c|c|c|c|c|c|}
\hline \multicolumn{8}{|c|}{ 2014-2015 } \\
\hline \multicolumn{4}{|c|}{ Premier cycle } & \multicolumn{4}{|c|}{ Second cycle } \\
\hline \multicolumn{2}{|c|}{ Scolarisation } & \multicolumn{2}{|c|}{ Achèvement } & \multicolumn{2}{|c|}{ Scolarisation } & \multicolumn{2}{|c|}{ Achèvement } \\
\hline Garçons & Filles & Garçons & Filles & Garçons & Filles & Garçons & Filles \\
\hline $74,8 \%$ & $63,4 \%$ & $53,8 \%$ & $45,7 \%$ & $52,9 \%$ & $47,8 \%$ & $32 \%$ & $24,2 \%$ \\
\hline \multicolumn{8}{|c|}{$2015-2016$} \\
\hline Garçons & Filles & Garçons & Filles & Garçons & Filles & Garçons & Filles \\
\hline $75 \%$ & $64,3 \%$ & $49,4 \%$ & $43 \%$ & $55,5 \%$ & $43,7 \%$ & $32,1 \%$ & $23,8 \%$ \\
\hline \multicolumn{8}{|c|}{$2016-2017$} \\
\hline Garçons & Filles & Garçons & Filles & Garçons & Filles & Garçons & Filles \\
\hline $75,8 \%$ & $64,4 \%$ & $48,8 \%$ & $41,3 \%$ & $55,6 \%$ & $46,8 \%$ & $33,2 \%$ & $26,9 \%$ \\
\hline
\end{tabular}

Source : Ministère de l'éducation nationale du Mali. 2018 (Loua, 2018)

Dans ce tableau, on peut remarquer que les effectifs des filles au niveau des taux de scolarisation et d'achèvement des cycles, sont toujours inférieurs à ceux des garçons. Avec les mesures prises pour améliorer la scolarisation des filles, la disparité entre garçons et filles au niveau du premier cycle de l'enseignement fondamental s'atténue progressivement. Les taux bruts de scolarisation des garçons et des filles étaient 
respectivement de $75,7 \%$ et $73,2 \%$ en $2018-2019$. Concernant le taux net de scolarisation sur la même période, cet écart est encore plus réduit entre garçons et filles avec respectivement $57,9 \%$ et $57,5 \%$ (EMOP, 2018).

Cependant, l'écart commence à se creuser au niveau du second cycle de l'enseignement fondamental, car les taux de fréquentation à ce niveau sont de 54,00\% pour les garçons et $53,7 \%$ pour les filles. Il faut reconnaître que malgré cet écart bien visible, les garçons connaissent plus de redoublement que les filles avec respectivement $5,2 \%$ et $5,1 \%$ au fondamental 1 qui correspond au premier cycle de l'enseignement fondamental d'une durée de six ans et $13,8 \%$ et $12,1 \%$ au fondamental 2 qui correspond au second cycle de l'enseignement fondamental d'une durée de trois ans. Ces chiffres sur les redoublements montrent que le décrochage scolaire des filles est lié à d'autres facteurs qui ne relèvent pas systématiquement de l'école.

L'écart entre garçons et filles s'accentue au niveau de l'enseignement secondaire malien avec respectivement 59,91\%,40,09 pour l'enseignement secondaire général ; $58,40 \%$ et $41,60 \%$ pour l'enseignement secondaire technique $; 59,27 \%$ et $40,73 \%$ pour l'enseignement secondaire professionnel $; 73,87 \%$ et $26,13 \%$ pour les Instituts de Formation des Maîtres (CPS/MEN, 2016).

Concernant l'enseignement supérieur, l'effectif des garçons est nettement supérieur à celui des filles. Les tableaux suivants font état de quelques statistiques des effectifs dans l'enseignement supérieur malien.

Tableau 2 : effectifs de l'enseignement supérieur sur deux ans

\begin{tabular}{|l|l|l|l|l|l|}
\hline \multirow{2}{*}{ Sexe } & \multicolumn{2}{|l|}{$2015-2016$} & \multicolumn{2}{l|}{$2016-2017$} & \multirow{2}{*}{$\%$} \\
\cline { 2 - 5 } & Effectif & $\%$ & Effectif & $\%$ & d'évolution \\
\hline F & 17137 & 29,10 & 20504 & 29,63 & 19,65 \\
\hline
\end{tabular}


The Journal of Quality in Education (JoQiE) Vol.11, Nº17, May 2021

\begin{tabular}{|l|l|l|l|l|l|}
\hline$M$ & 41743 & 70,90 & 48698 & 70,37 & 16,66 \\
\hline Total & 58880 & 100 & 69202 & 100 & 17,53 \\
\hline
\end{tabular}

Source : Direction Générale de l'Enseignement Supérieur et de la Recherche Scientifique, 2017

Tableau3 : effectifs par sexe et année d'études supérieures

\begin{tabular}{|l|l|l|l|}
\hline \multirow{2}{*}{ Année d'études } & \multicolumn{2}{|l|}{ Sexe } & \multirow{2}{*}{ Total général } \\
\cline { 2 - 3 } & F & M & \\
\hline 1 & 9759 & 21603 & 31362 \\
\hline 2 & 4324 & 10478 & 14802 \\
\hline 3 & 2743 & 7286 & 10029 \\
\hline 4 & 3190 & 7694 & 10884 \\
\hline 5 & 220 & 825 & 1045 \\
\hline 6 & 196 & 506 & 702 \\
\hline 7 & 72 & 306 & 378 \\
\hline Total général & 20504 & 48698 & 69202 \\
\hline
\end{tabular}

Source : Direction Générale de l'Enseignement Supérieur et de la Recherche Scientifique, 2017

Dans ces tableaux, nous constatons que la disparité entre filles et garçons s'accentue d'avantage au niveau de l'enseignement supérieur. Même si les effectifs des filles progressent mieux, ils représentent moins du tiers de l'effectif total. Dans le tableau 3, nous voyons que plus le niveau d'études augmente, plus le décrochage est patent. 
The Journal of Quality in Education (JoQiE) Vol.11, Nº17, May 2021

\section{Principaux facteurs de décrochage scolaire des filles}

Le décrochage scolaire des filles au Mali peut être lié à plusieurs facteurs émanent des traditions et cultures familiales, à l'insécurité que le pays connaît depuis 2012, mais aussi à l'environnement interne de l'école. Selon un auteur (Purcell, 1995) « Il existe, dans le monde entier, des preuves de discrimination et de violence à l'égard des filles, qui commencent bien avant leur naissance et continuent sans relâche tout au long de leur existence. Durant l'enfance et l'adolescence, les filles ont souvent un accès plus limité à la nutrition, à la santé physique et mentale et à l'éducation, et moins de droits, de chances et de possibilités que les garçons. Il n'est pas rare qu'elles fassent l'objet d'une exploitation sexuelle et économique, de pratiques de violences et préjudiciables, telles que sélection prénatale du sexe, infanticide, mutilations génitales et mariages précoces ».

\section{Situations économiques défavorables}

$\mathrm{Au}$ Mali l'enseignement fondamental public est officiellement gratuit, cependant, selon un chapitre d'ouvrage (Marcoux, 1998) « les parents doivent encore assurer un certain nombre de dépenses pour que leurs enfants puissent fréquenter l'école dans des conditions propices à la réussite. Il s'agit, tout d'abord, des dépenses vestimentaires, d'achats de livres ou de matériel pédagogique. Mais aussi, dans les écoles communautaires, des contributions des parents au fonctionnement de l'école. Enfin, à cause des conditions pédagogiques loin d'être optimales (surcharge des classes, démotivation des enseignants, etc.) ils doivent, à défaut de le faire eux-mêmes, payer pour les soutiens scolaires privés. Face à toutes ces dépenses nécessaires sinon indispensables pour améliorer la réussite scolaire, la faiblesse du niveau de vie de la famille constitue un handicap ». En cas de nécessité, le parent préfère investir dans 
l'éducation du garçon plutôt que celle de la fille, car cette dernière est appelée à se marier et à être théoriquement à la charge d'un homme dans son foyer. Le même auteur poursuit en disant ceci «La réussite scolaire repose sur la possibilité pour l'élève à se concentrer sur ses études, sans être distrait par d'autres activités (domestiques, économiques). Or pour que l'élève ne se consacre qu'à ses études, il faut que le ménage auquel il appartient puisse fonctionner sans sa contribution régulière aux différentes activités domestiques et économiques. Cette condition va être difficile à satisfaire dans les ménages les plus pauvres, où les enfants constituent une main- d'œuvre par excellence soit pour les tâches domestiques, soit pour les activités économiques ». Au Mali, en 2019, il a été remarqué que 13,3\%, contre 7,3\% de garçons ne fréquentent pas le premier de l'enseignement fondamental à cause du manque de moyen financier des parents (EMOP, 2018). Selon la même source le taux de chômage des 15-64 ans au Mali en 2018 était estimé à 9,7\% pour les femmes et 7,5\% pour les hommes. La proportion d'individus pauvres dans la population malienne était estimée à 44,9\% au niveau national, avec 4,7\% à Bamako (la capitale) et 53,6\% en milieu rural en 2017 (INSTAT, 2018). Cette pauvreté à un impact sur la scolarisation des filles, notamment en milieu rural où vivent environ $74 \%$ de la population malienne, car en cas de difficultés financières, les familles préfèrent scolariser le garçon qui est appelé à devenir chef de foyer et à déscolariser la fille qui est supposée être prise en charge par son mari.

\section{Tâches domestiques}

Au Mali, notamment dans le milieu urbain, la jeune fille est appelée à participer régulièrement aux travaux domestiques en dehors des heures de cours, ce qui peut réduire considérablement son temps d'apprentissage et jouer par ricochet sur son rendement scolaire. Au temps colonial, la réticence à la scolarisation des enfants en général était manifeste chez les parents, pour les filles, cette réticence était encore plus 
élevée à cause des tâches domestiques. Dans sa thèse de doctorat, (Traoré, 2009), explique que la femme est tenue simplement d'entretenir le foyer, parce que le statut physique de la fille, la prédispose à certaines activités. Elle ne doit point s'en dérober. Selon Marie France Lange (2009) « Le rôle de mère et d'épouse dévolu à ces dernières serait la raison de leur plus grande implication aux travaux domestiques. Aussi doiventelles apprendre très tôt à concilier l'école et le travail, ce qui semble plus difficile au début de la scolarité ». L'éducation traditionnelle dans les familles maliennes, pendant que le garçon suit et aide son père dans des activités supposées être masculines, la fille, avec sa mère s'occupent des activités à connotation féminine. Dans un article sur la situation éducative des filles et des femmes au Mali (Loua, 2018), il ressort que l'homme étant d'emblée chef de famille, est chargé de la recherche du gain quotidien, tandis que la femme est appelée à s'occuper des activités au sein de la famille relevant du ménage et de l'entretien des enfants. La reproduction sociale du rôle de future épouse de la fille commence dès à l'adolescence, bien qu'elle soit scolarisée. La fréquence des travaux domestiques a donc une incidence sur le rendement scolaire des filles, ce qui se manifeste par l'inattention en classe, l'absentéisme, la fatigue, les retards récurrents, etc., qui peuvent déboucher sur le décrochage scolaire à l'interne ou vers l'extérieur qui engendre l'abandon scolaire définitif.

\section{L'insécurité}

Selon une étude (OCHA, 2018) 2 millions d'enfants n'ont pas accès à l'école, dont 220 500 subissent les conséquences directes du conflit, qui a entrainé la fermeture de 735 écoles dans les régions centre et nord du Mali. Avec 735 écoles toujours fermées, l'offre éducative est insuffisante et entraine la déscolarisation et la non-scolarisation de milliers d'enfants y compris des jeunes filles ». Selon la même étude, « dans les écoles ouvertes, l'absence de cantines scolaires est un facteur limitant pour assurer le maintien et la 
fréquentation constante des enfants à l'école à Kidal, Ménaka et dans certaines zones de Gao et Tombouctou ». Ce phénomène d'absence de cantines scolaires, qui peut engendrer le décrochage, notamment à cause de la distance à parcourir pour se rendre à l'école dans certaines localités rurales, touche les filles plus que les garçons. Le terrorisme qui s'est installé au Mali depuis 2012 a fortement impacté l'école. Des groupes terroristes comme le Front de Libération du Macina (FLM), et les bandits armés qui s'insurgent contre l'école occidentale, sont particulièrement contre la scolarisation des filles. Les filles sont donc fondamentalement touchées par l'insécurité dans les régions du nord et celles du centre du Mali, car elles sont victimes d'enlèvements et de viols. Selon l'enquête auprès des ménages (EMOP, 2019), 36,4\% des filles ne fréquentent pas le premier cycle de l'enseignement fondamental à cause de la crise sécuritaire au Mali.

\section{Mariage d'enfants et grossesses précoces}

Au Mali l'âge minimum pour contracter le mariage est de dix-huit ans pour l'homme et seize-ans pour la femme selon le code des personnes et de la famille de décembre 2011. Cependant, dans le milieu rural, cet âge minimum n'est pas toujours respecté concernant les filles. Selon Plan international, cité dans un article (Loua, 2018), « en Afrique, plus de $50 \%$ de filles donnent naissance à leur premier enfant avant l'âge de 21 ans. La grossesse précoce entraîne souvent un mariage précoce, pour préserver l'honneur de la famille dans un milieu où la population reste profondément ancrée dans sa culture ». Au Mali, entre 2006 et 2011, 50,4\% des mariages concernaient les filles âgées de 15 à 19 ans et $20 \%$ de ces filles vivaient dans des familles polygames (Walker, 2013). Selon l'Enquête démographique et de santé au Mali (2012-2013), « une femme sur cinq (20\%) était déjà en union avant d'atteindre l'âge de 15 ans. La moitié des femmes (50\%) était déjà en union avant 18 ans, près de deux adolescentes sur cinq 
(39\%) ont déjà commencé leur vie reproductive et $33 \%$ ont eu, au moins, un enfant ». Avec le poids des activités familiales et la prise en charge d'un nouveau-né, la jeune fille mariée peut être facilement exposée au décrochage scolaire précoce. Selon l'enquête modulaire auprès des ménages (EMOP, 2019), 33,4\% des filles abandonnent le second cycle de l'enseignement fondamental au Mali à cause des mariages et grossesses précoces.

\section{Climat scolaire}

Le climat scolaire que nous pouvons qualifier de climat relationnel, éducatif, de sécurité, de justice, de violence, d'indiscipline, d'accessibilité aux drogues, les systèmes d'encadrement, de qualité de l'enseignement, de politique pédagogique, etc. ; s'il est négatif peut être facteur de décrochage scolaire, notamment pour les filles. Les écoles maliennes (fondamentales, secondaires et supérieures), sont régulièrement émaillées de violences verbales et physiques. Ces violences sont visibles particulièrement au niveau de l'enseignement supérieur où les renouvellements de bureaux de l'Association des Elèves et Etudiants du Mali (AEEM) donnent toujours lieu à des actes d'agressions. La mort de trois étudiants maliens en 2018 et 2019 sous les coups de leurs camarades constitue un témoignage éloquent à cet effet. Dans un article, Idrissa Soïba Traoré (2008), dit que « la mise en place des bureaux de l'AEEM constitue les manifestations les plus patentes de la violence. Ces violences donnent lieu à des séquestrations, des menaces, des échauffourées entre les différents acteurs. Tout cela se fait souvent au prix d'un saccage complet des infrastructures publiques ». Pierre Potvin (2004), identifie le climat de classe (gestion de classe) et la relation enseignant-élève (pratiques pédagogiques favorisant l'engagement de l'élève) qui sont associés au risque du décrochage scolaire. 
The Journal of Quality in Education (JoQiE) Vol.11, N²17, May 2021

\section{Mesures d'amélioration de la situation scolaire des filles au Mali}

La sensibilisation des acteurs de l'éducation, l'engagement des parents et des responsables religieux sont nécessaires pour la protection des filles contre les mariages des enfants et contre les violences dont les filles sont régulièrement victimes dans les établissements scolaires et universitaires. De l'indépendance du Mali en 1960 à nos jours, plusieurs mesures, notamment des réformes et innovations pédagogiques, ont été adoptées par les autorités politiques et scolaires maliennes, en collaboration avec les partenaires de l'éducation pour relever le défi de la scolarisation des filles ainsi que leur maintien à l'école jusqu'à la fin de la scolarité obligatoire ou à l'obtention d'un diplôme. La scolarisation des filles et leur maintien à l'école était dans l'esprit de toutes les grandes réformes et innovations pédagogiques maliennes. La réforme de l'enseignement de 1962 avec son objectif 'une éducation de masse et de qualité', la Nouvelle Ecole Fondamentale en 1994 et le Programme Décennal de Développement de l'Education de 1998 avec son axe référentiel 'un village une école', voulaient améliorer la situation scolaire malienne en général et celle des filles en particulier qui étaient victimes de discrimination depuis l'époque coloniale. Dans sa thèse de doctorat (Diallo, 2001), évoque certaines mesures en ces termes « plusieurs Projets de Développement de l'Éducation de Base (PDEB) avaient été initiés, dont celui de l'UNESCO en 1972 qui encourageait la scolarisation des filles tant en milieu urbain qu'en milieu rural. Toujours dans le cadre de la mise en œuvre des Projets de Développement de l'Éducation de Base, la Banque Mondiale, AID et le Gouvernement malien ont inscrit, en 1990, un projet dont les composantes étaient la scolarisation et la rétention des filles à l'école, appelé Cellule Nationale de Scolarisation des Filles (CNSF). La première étape du volet scolarisation des filles a consisté à sensibiliser les 
parents à la promotion et à la nécessité de l'éducation des filles à travers les médias ». Dans un article (Loua, 2018), certaines mesures sont évoquées, notamment la mise en place, en 1992, de la Cellule nationale de scolarisation des filles et de ses antennes dans huit régions à l'époque, avait pour but de tenir compte des spécificités régionales et locales et de favoriser une meilleure implication des partenaires de l'école dans la promotion de la scolarisation des filles. La création en 2000 de la Section scolarisation et formation des filles et la mise en place en 2011 de la Division de la scolarisation des filles, pour la promotion de la scolarisation et du maintien des filles à l'école. La politique nationale de l'alimentation scolaire, à travers des cantines dans certaines écoles fondamentales situées à plus de cinq kilomètres des domiciles dans le milieu rural, soutenue par les mairies, les familles et les partenaires, est en cours pour maintenir les filles à l'école. Une politique d'encouragement des activités génératrices de revenus, comme le maraîchage, la couture, la teinture, le commerce, etc. Cette politique a été mise en place à l'endroit des femmes pour réduire le travail des enfants et favoriser le maintien des filles à l'école. Les centres de formation professionnelle, dont celle du quartier de Lassa créée en 1998 et celle de Missabougou créée en 2006, œuvrent dans ce sens.

En plus de ces mesures, qui ont permis une certaine amélioration de la situation scolaire des filles au Mali, l'intensification des campagnes de sensibilisation pour éveiller les consciences sur les conséquences des mariages et grossesses d'enfants, à travers l'implication des responsables religieux et coutumiers, est nécessaire dans les zones rurales afin d'accroître d'avantage la scolarisation des filles et leur maintien à l'école. Certaines Organisations Non Gouvernementales, comme l'ONG Agir et Save the children, travaillent dans ce sens pour assurer l'accès aux services de santé et réduire les grossesses précoces des élèves dans la région de Sikasso au Mali. 


\section{Conclusion}

Le décrochage scolaire des filles au Mali peut être globalement lié au capital économique des familles, d'où la capacité des ménages à assurer les besoins en dépenses de l'éducation; le capital culturel qui fait allusion à la connaissance de l'importance de l'éducation de la part des parents ainsi que leur accompagnement dont l'enfant doit bénéficier durant son parcours scolaire. L'environnement familial peut être un facteur d'échec scolaire notamment les travaux domestiques dans lesquels les filles sont régulièrement impliquées.

Le renforcement de la législation qui interdit le mariage d'enfants de moins de seize ans doit être une priorité des autorités afin d'éviter le mariage de la fille avant la fin de scolarité obligatoire. Les autorités scolaires, à travers les collectivités territoriales, devront s'atteler à la mise en place du cycle complet de l'enseignement fondamental (9 ans) dans les milieux ruraux où l'offre d'éducation est insuffisante, afin d'éviter les longues distances à parcourir et les changements de localités qui peuvent engendrer l'abandon scolaire des filles. Les mauvais traitements dans les familles d'accueil, les grossesses que les filles peuvent contracter loin de la surveillance des parents sont des facteurs de décrochage scolaire.

\section{Bibliographie}

Cellule de Planification et de Statistique/Ministère de l'éducation nationale (2017). Annuaire nationale des statistiques des enseignements secondaire et normal 2015-2016. CPS/MEN.

Institut National de la Statistique du Mali (2018). Enquête modulaire et permanente auprès des ménages. (EMOP). INSTAT/Rapport d'analyse premier passage, Août 2018. 75p

Institut National de la Statistique du Mali (2017). Enquête modulaire et permanente auprès des ménages. (EMOP). INSTAT/Rapport d'analyse premier passage. $76 \mathrm{p}$. 
MARCOUX, R. (1998). Entre l'école et la calebasse. Sous-scolarisation des filles et mise au travail à Bamako. in: LANGE M.-F. (1998). L'école et les filles en Afrique. Scolarisation sous condition. Paris, Karthala, 1998, p. 73-96.

LOUA, S. Etat des lieux de l'éducation de filles et des femmes au Mali : contraintes et défis. Revue internationale d'éducation de Sèvres, n78/2018, p 103-113.

OCHA (2019). Aperçu des besoins humanitaires. OCHA/Mali. 45 p.

OPHEIM, M., (2000). Les filles et l'école au Mali. Université d'Olso/Norvège/Nordic Journal of African Studies. P 152-171.

POTVIN, P., et al (2004). Guide de prévention du décrochage scolaire. Québec, Centre de transfert pour la réussite éducative du Québec.

PURCELL, M. (1995). Inscrire les filles à l'ordre du jour. in Les Enfants d'abord. Journal de l'UNICEF (3) New York: UNICEF.

TOUNKARA, A., (2015). Femme et discrimination au Mali. Paris, l'Harmattan, 218 p.

TRAORE I. S. (2008) La crise scolaire au Mali, fille de la crise des valeurs sociales. Revue UCAO n ${ }^{\circ} 0$

TRAORE I. S. (2009). Education et décentralisation au Mali: enjeux et réalités des dynamiques d'appropriations locales (cas des communes de Dombila, Markala, Kati et commune V du District de Bamako. Thèse de doctorat, Université Paris 8 Vincennes, Saint-Denis/Département des Sciences de l'éducation. 462 p.

UNESCO (2015). Examen national 2015 de l'éducation pour tous (EPT) : Mali.

UNESCO/Ministère de l'Education Nationale. 29 p 